\title{
Comparing the host galaxies of different type supernovae
}

\author{
Y. C. Liang ${ }^{1}$, X. Shao ${ }^{1,3}$, M. Dennefeld ${ }^{2}$, X. Y. Chen ${ }^{1}$, \\ L. Zhou ${ }^{1}$ and F. Hammer ${ }^{3}$
}

\author{
${ }^{1}$ Key Laboratory of Optical Astronomy, National Astronomical Observatories, Chinese \\ Academy of Sciences, 20A Datun Road, Chaoyang District, Beijing, 100012, China \\ ${ }^{2}$ Institut d'Astrophysique de Paris, CNRS, and Universite P. et M. Curie, 98bis Bd Arago, \\ F-75014 Paris, France \\ ${ }^{3}$ Laboratoire GEPI,Observatoire de Paris, CNRS-UMR8111, Univ. Paris Diderot, Sorbonne \\ Paris Cité, 5 place Jules Janssen, F-92195 Meudon, France
}

\begin{abstract}
We compare the host galaxies of 902 supernovae, including Type Ia, II and Ibc, which are selected by cross-matching the Asiago Supernova Catalog with the SDSS DR7. We further selected 213 galaxies by requiring the light fraction of spectral observations $>15 \%$, which could represent well the global properties of the galaxies. The diagrams related to $\mathrm{D}_{n}(4000), \mathrm{H} \delta_{A}$, stellar masses, SFRs and specific SFRs for the SNe hosts show that almost all SNe II and most of SNe Ibc occur in SF galaxies. A significant fraction of SNe Ia occurs in AGNs and Absorp galaxies. These results are compared with those of the 689 comparison galaxies where the SDSS fiber captures $<15 \%$ of the total light. These comparison galaxies appear biased towards higher $12+\log (\mathrm{O} / \mathrm{H})(\sim 0.1 \mathrm{dex})$ at a given stellar mass, suggesting the aperture effect should be kept in mind when the properties of the hosts for different types of SNe are discussed.
\end{abstract}

Keywords. galaxies: evolution, galaxies: star formation, galaxies: starburst

Cross-correlations between ASC (Barbon et al. 1999) and SDSS-DR7 MGS (Abazajian et al. 2009) using 15 arcsec radius are made to select the supernova host galaxies and we got 902 host galaxies. Among them, 213 host galaxies have light fraction ( $l f$, Liang et al. 2010) more than 15\%, denoting that their 3 arcsec fiber observation by SDSS cover most light of the whole galaxies. By comparing the relations between $\mathrm{D}_{n}(4000), \mathrm{H} \delta_{A}$, stellar masses, SFRs and specific SFRs, we found that almost all the SNe II and most SNe Ibc occur in star forming (SF) galaxies (having a wide range of stellar mass and low $\left.\mathrm{D}_{n}(4000)\right)$. And only very few are in the AGNs and weak emission-line \& Absorption galaxies (massive and have high $\mathrm{D}_{n}(4000)$ ). The majority of the SNe Ia occur in AGNs and absorption-line galaxies and about one-third in SF galaxies. The K-S test for stellar population analysis shows that the hosts of SNe II are younger than the hosts of type Ia SNe. The hosts of SNe Ia are more metal-rich than hosts of SNe II. When compared to $689 \mathrm{SN}$ host galaxies with $l f<15 \%$, these 213 galaxies appear biased towards lower $12+\log (\mathrm{O} / \mathrm{H})(\sim 0.1 \mathrm{dex})$ at a given stellar mass, suggesting the aperture effect should be kept in mind when the properties of the hosts for different types of SNe are discussed.

\section{Acknowledgements}

This work was supported by the Natural Science Foundation of China (NSFC) Foundation under No. 11273026.

\section{References}

Abazajian, K. N., Adelman-McCarthy, J. K., Agüeros, M. A., et al. 2009, ApJS, 182, 543

Barbon, R., Buondí, V., Cappellaro, E., \& Turatto, M. 1999, A\&AS, 139, 531

Liang, Y. C., Zhong, G. H., Hammer, F., et al. 2010, MNRAS, 409, 213 